Archive for

Organic Chemistry

Arkivoc 2018, part vii, 423-430

\title{
Selective synthesis of methyl dithienyl-glycolates
}

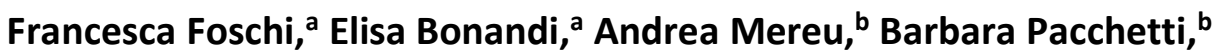 Davide Gozzini, ${ }^{b}$ and Daniele Passarella*a}

aDipartimento di Chimica, Università degli Studi di Milano, Via Golgi 19, Milano 20133, Italy

${ }^{b}$ LINNEA SA, Via Cantonale, 6595 Riazzino (TI), Switzerland

Email: daniele.passarella@unimi.it

Received 08-06-2018

Accepted 10-26-2018

Published on line 12-01-2018

\section{Abstract}

An efficient selective synthesis of methyl dithienyl-glycolates has been developed. The interest of this two steps protocol resides in the possibility of synthesized either methyl 2,2-dithienyl glycolate - the target intermediate for the preparation of anticholinergic agents - or its regio-isomer methyl 2,3-dithienyl glycolate the most critical precursor of anticholinergic drug impurity.

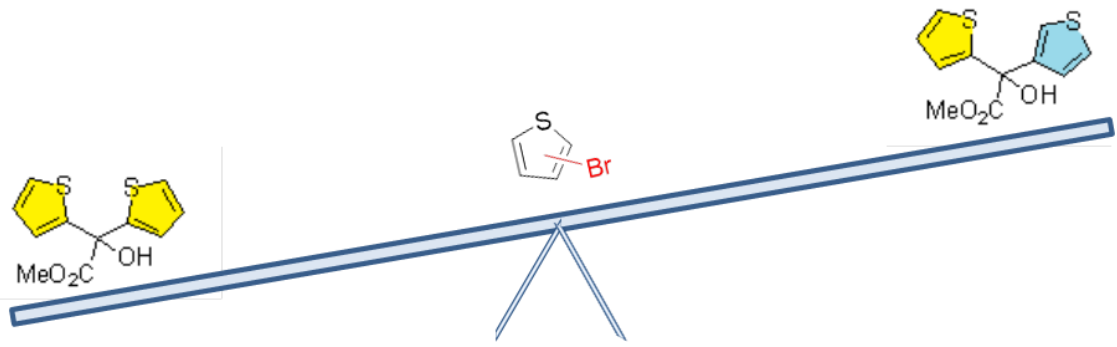

Keywords: Bromothiophene lithiation, dithienyl glycolate, muscarinic antagonist, COPD 


\section{Introduction}

Methyl 2,2-DiThienylGlycolate (1a, Figure 1) is a key intermediate for the preparation of a wide range of anticholinergic agents, as muscarinic receptor antagonists, used in the treatment of 'Chronic Obstructive Pulmonary Disease' (COPD). In particular, condensation of 1a with scopine or 3-quinuclidinol derivatives under basic conditions, followed by quaternization of the tertiary amino moiety to increase their lipophilicity, is a classical pharmaceutical process to obtain tiotropium bromide (Scheme 1; marketed as SPIRIVA ${ }^{\circledR}$ HANDIHALER $\left.{ }^{\circledR}\right),{ }^{1-7}$ aclidinium bromide (trade name: TUDORZA $^{\circledR}$ PRESSAIR $^{\circledR}$ ), ${ }^{8}$ and their analogues. ${ }^{9-18}$

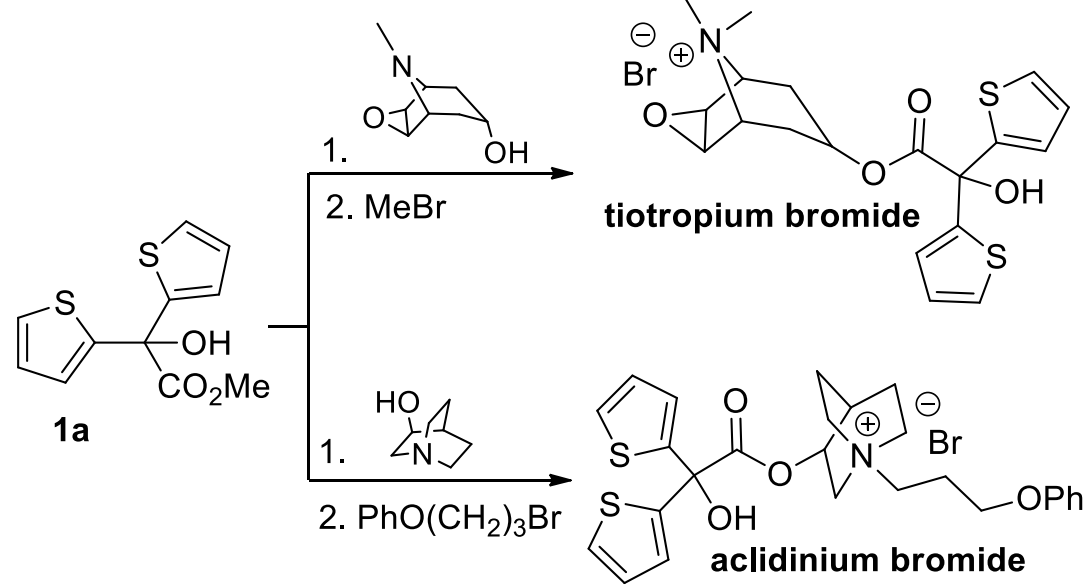

Scheme 1. Synthesis of tiotropium bromide and aclidinium bromide starting from 1a.

In general, synthesis of $1 \mathbf{a}$ is carried out via either Friedel-Crafts acylation of thiophene, ${ }^{19-29}$ or Grignard reactions. ${ }^{30}$ In our hands, reaction of the Grignard reagent derived from 2 a with dimethyl oxalate 3 in diethyl ether at reflux afforded $1 \mathrm{a}$ in $54 \%$ yield, but in inseparable mixture with an impurity identified as the regioisomer $\mathbf{1 b}$ (12\% yield, Scheme 2); $\mathbf{1 b}$ probably derived by equilibration of the initial 2-thienylmagnesium bromide.

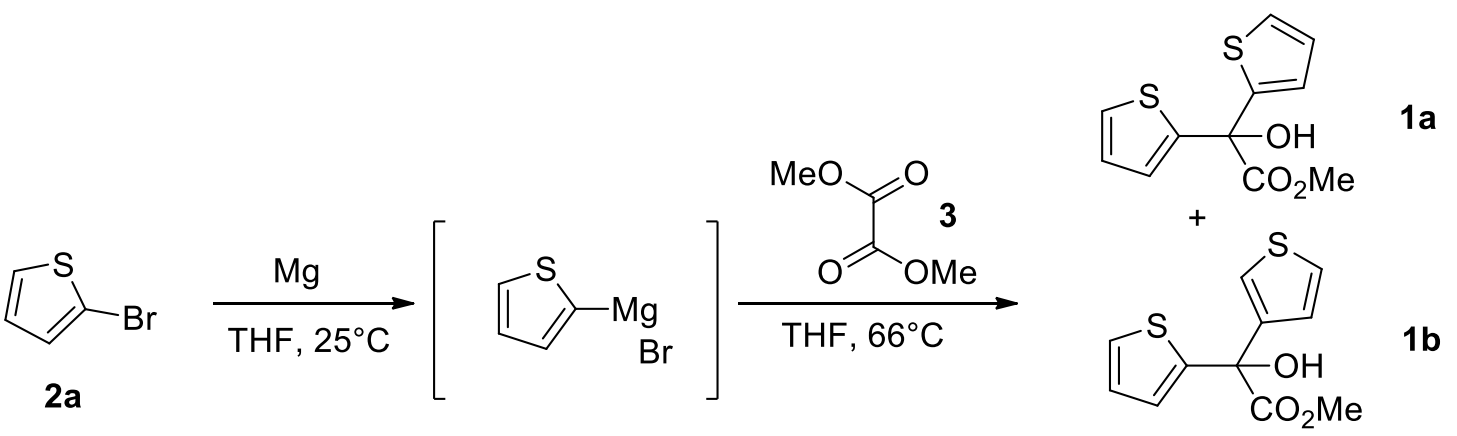

Reaction conditions: $2 \mathrm{a}(2.0 \mathrm{mmol}), 3(1.0 \mathrm{mmol}), \mathrm{Mg}(2.1 \mathrm{mmol})$, solvent $(10 \mathrm{~mL})$.

Scheme 2. Reported synthesis of dithienylglycolate $\mathbf{1 a - b}{ }^{[a]}$. 


\section{Results and Discussion}

The difficult separation of $\mathbf{1 a}$ and $\mathbf{1 b}$ leads to pollute drugs, due to the formation of pharmacological regioisomer impurities. ${ }^{31}$ Thus, to find an alternative method to selectively obtain either $\mathbf{1 a}$ or $\mathbf{1 b}$, we decided to modulate the reactivity of the thienyl anion species by changing the nature of the counter cation. To that aim 2- thienyl lithium and 3- thienyl lithium (derived, respectively, from lithiation of 2-bromothiophene 2a and 3bromothiophene $\mathbf{2 b}$ ), were reacted with oxalate $\mathbf{3}$ to obtain methyl 2-oxo-2-(thiophen-2-yl)acetate $\mathbf{4 a}$ and methyl 2-oxo-2-(thiophen-3-yl)acetate $\mathbf{4 b}$. The latter compounds are intermediates for methyl dithienylglycolate $\mathbf{1 a}$ and $\mathbf{1 b}$ production. Preliminary runs (entries 1-2, Table 1), evidenced that a mixture of regioisomers $\mathbf{4 a}$ and $\mathbf{4 b}$ could be obtained when oxalate $\mathbf{3}$ was added to 3 -thienyl lithium: in according to the anion equilibration, the longer the metalation time of $\mathbf{2 b}$, the higher the yield of $\mathbf{4 a}$.

Table 1. Reactivity of bromothiophene $\mathbf{2} \mathbf{b}^{\text {a }}$

\begin{tabular}{|c|c|c|c|c|c|}
\hline 2b $\mathrm{Br}$ & & $\begin{array}{l}\text { Solvent } \\
\text { in, }-78^{\circ} \mathrm{C}\end{array}$ & $\mathrm{O}^{\prime 4}$ & & $\mathrm{CO}_{2} \mathrm{Me}$ \\
\hline Entry & Base & solvent & Base addition & $4 a \%^{b}$ & $4 \mathrm{~b} \% \mathrm{~b}$ \\
\hline 1 & BuLi & THF & A & 35 & 24 \\
\hline 2 & BuLi & THF & B & 61 & -- \\
\hline 3 & BuLi & THF & C & -- & 48 \\
\hline 4 & BuLi & THF & D & -- & 66 \\
\hline 5 & BuLi & $\mathrm{Et}_{2} \mathrm{O}$ & D & -- & 35 \\
\hline 6 & BuLi & Toluene & D & -- & 22 \\
\hline 7 & LDA & THF & D & $<5$ & -- \\
\hline
\end{tabular}

${ }^{a}$ Reaction conditions: 3 (1.5 mmol), 2 b $(1 \mathrm{mmol})$, Base $(0.95 \mathrm{mmol})$, solvent $(10 \mathrm{~mL}) .{ }^{\mathrm{b}}$ Isolated yields.

A: Base was dropped in 5 min to a solution of $\mathbf{2 b}$ and stirred for $15 \mathrm{~min}$ before addition of a solution of $\mathbf{3}$; $\mathbf{B}$ : Base was dropped in $5 \mathrm{~min}$ to a solution of $\mathbf{2} \mathbf{b}$ and stirred for $30 \mathrm{~min}$ before addition of a solution of $\mathbf{3}$; $\mathbf{C}$ : $\mathbf{2} \mathbf{b}$ was added to a solution of BuLi and $\mathbf{3}$ in THF; D: Base was added to a solution of $\mathbf{2} \mathbf{b}$ and $\mathbf{3}$.

In fact, a complete regio-selectivity in favor of $\mathbf{4 b}$ was reached by adding $\mathbf{2 b}$ to a cold mixture of base ( $n$ BuLi) and $\mathbf{3}$ (entry 4$)$. Furthermore, the best yield of $\mathbf{4 b}(66 \%$, entry 4$)$ was achieved by adding the strong, nonhindered base $n$-BuLi at $-78{ }^{\circ} \mathrm{C}$, to a mixture of $\mathbf{2 b}$ and a slightly excess of $\mathbf{3}$ in THF as the solvent. Other solvents (entries 5-6), gave worst results while, the use of non-nucleophilic base LDA, resulted in the formation of a series of by-products (entry 7). Similarly, LDA induced on 2a a base catalyzed halogen-dance reaction, forming, as the main reactive intermediate, the 2 - thienyl anion specie bearing the bromine atom in the C-3 position. ${ }^{32-36}$ This latter, in turn, evolves in the $\mathbf{4 c}$ product in presence of 3 (entry 1, Table 2). Furthermore, $\mathbf{1 a}$ reacted under the best reaction conditions found for $\mathbf{1 b}$, giving $\mathbf{4 a}$ in good yield (entry 2); we were able to increase the yield of $4 \mathrm{a}$ by generating the stable lithium anion at the thiophene $\mathrm{C}-2$ position and then adding oxalate $\mathbf{3}$ to the reaction mixture (entry 3 ). 
Table 2. Reactivity of bromothiophene $2 a^{a}$

\begin{tabular}{|c|c|c|c|c|c|}
\hline $2 a$ & & $\begin{array}{l}\text { e, Solvent } \\
\text { in, }-80^{\circ} \mathrm{C}\end{array}$ & $\mathrm{O}_{4 a}^{\mathrm{T}}$ & & $-\mathrm{CO}_{2} \mathrm{~N}$ \\
\hline Entry & Base & solvent & Base addition & $4 a \%^{b}$ & $4 c \%^{b}$ \\
\hline 1 & LDA & THF & A & -- & 36 \\
\hline 2 & BuLi & THF & A & 67 & -- \\
\hline 3 & BuLi & THF & B & 73 & -- \\
\hline
\end{tabular}

a Reaction conditions: 3 (1.5 mmol), 2b $(1 \mathrm{mmol})$, Base $(0.95 \mathrm{mmol})$, solvent $(10 \mathrm{~mL}) .{ }^{\mathrm{b}}$ Isolated yields. A: Base was added to the mixture of $\mathbf{2 a}$ and 3; B: Base was dropped in $5 \mathrm{~min}$ to a solution of $\mathbf{2 a}$ and stirred for $30 \mathrm{~min}$ before addition of a solution of $\mathbf{3}$.

Similar behavior (Scheme 3) was found when the 2-thienyl anion specie, generated by metalation of $2 \mathbf{a}$ at low temperature, was then trapped using as electrophiles $\mathbf{4 a}$ or $\mathbf{4 b}$ : under these reaction conditions, compounds $\mathbf{1 a}$ (derived from intermediate $\mathbf{4 a}$, path a.) and $\mathbf{1 b}$ (derived from $\mathbf{4 b}$, path $\mathbf{b}$.) were isolated as pure isomers and fully characterized.

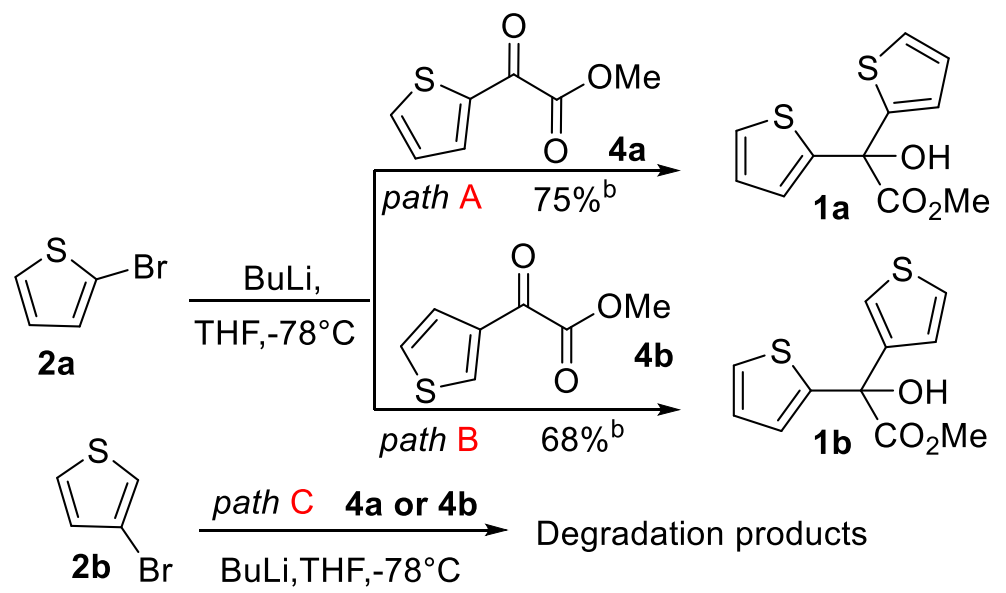

a Reaction conditions: 2 (1.0 mmol), 4 (1.0 mmol), Base $(0.95 \mathrm{mmol})$, solvent $(10 \mathrm{~mL})$, reaction time: $30 \mathrm{~min} .{ }^{\mathrm{b}}$ Isolated yields.

path A and B: BuLi was dropped in 5 min to $\mathbf{2 a}$ and the mixture was stirred for 30 min before addition of 4; path $\mathrm{C}$ : Base was added to the mixture of $\mathbf{2 b}$ and $\mathbf{4}$.

Scheme 3. Synthesis of $\mathbf{1 a}$ and $\mathbf{1 b} .^{\mathrm{a}}$

On the contrary, our attempts to isolate $\mathbf{1 b}$ or its regio-isomer methyl 3,3-dithienylglycolate by 3bromothiophene $\mathbf{2} \mathbf{b}$ lithium halogen exchange in the presence of either oxo-acetate $\mathbf{4 b}$ or $\mathbf{4 a}$, gave a mixture of degradation compounds (path c.). Comparison between ${ }^{1} \mathrm{H}$ NMR spectra of compounds $\mathbf{1 a}$ and $\mathbf{1 b}$ (Table 3 ), exhibited a slightly highfield chemical shifts for almost all $\mathbf{1 a}$ signals (only $\mathbf{1 a}$ and $\mathbf{1} \mathbf{b} \mathrm{H}_{3}$ protons resonate together at $7.19 \mathrm{ppm})$; furthermore, $\mathbf{1} \mathbf{b}^{1} \mathrm{H}$ NMR showed two additional signals at $7.39 \mathrm{ppm}\left(\mathrm{H}_{7}\right)$ and 7.10 
ppm $\left(\mathrm{H}_{9}\right)$. Even for ${ }^{13} \mathrm{C}$ NMR spectra, all the peaks of $\mathbf{1} \mathbf{a}$ and $\mathbf{b}$ are quite good shifted; the major difference arise in the presence on substrate $\mathbf{1} \mathbf{b}$ of both the isolated signal at $142.5 \mathrm{ppm}\left(\mathrm{C}_{6}\right)$ and $123.2 \mathrm{ppm}\left(\mathrm{C}_{7}\right)$.

Table 3. ${ }^{1} \mathrm{H}$ and ${ }^{13} \mathrm{C}$ assignment for $\mathbf{1 a}$ and $\mathbf{1 b}$

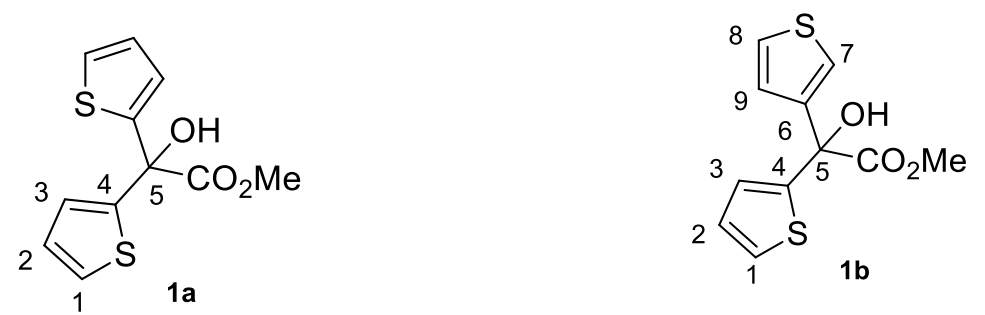

\begin{tabular}{|c|c|c|c|c|c|c|}
\hline${ }^{1} \mathrm{H}$ & ${ }^{13} \mathrm{C}$ & position & ${ }^{1} \mathrm{H}$ & ${ }^{13} \mathrm{C}$ & ${ }^{1} \mathrm{H}$ & ${ }^{13} \mathrm{C}$ \\
\hline \multicolumn{2}{|c|}{$\mathrm{CDCl}_{3}$} & & \multicolumn{2}{|c|}{$\mathrm{CDCl}_{3}$} & \multicolumn{2}{|c|}{$d_{6}$-Acetone } \\
\hline 7.32 & 126.0 & 1 & 7.28 & 125.8 & 7.41 & 126.1 \\
\hline 7.00 & 126.1 & 2 & 6.97 & 126.7 & 7.00 & 127.1 \\
\hline 7.19 & 126.8 & 3 & 7.18 & 125.9 & 7.12 & 126.2 \\
\hline-- & 145.7 & 4 & -- & 146.0 & -- & 147.9 \\
\hline-- & 76.6 & 5 & -- & 77.5 & -- & 77.5 \\
\hline 4.68 & -- & $\mathrm{OH}$ & 4.47 & -- & 5.76 & -- \\
\hline-- & 172.8 & CO & -- & 173.4 & -- & 173.3 \\
\hline \multirow[t]{5}{*}{3.92} & 54.2 & $\mathrm{OCH}_{3}$ & 3.87 & 53.9 & 3.83 & 53.3 \\
\hline & & 6 & -- & 142.5 & -- & 144.7 \\
\hline & & 7 & 7.39 & 123.2 & 7.47 & 123.2 \\
\hline & & 8 & 7.28 & 125.8 & 7.44 & 126.0 \\
\hline & & 9 & 7.10 & 126.8 & 7.22 & 127.6 \\
\hline
\end{tabular}

\section{Conclusions}

We have described a complete regio-selective protocol for methyl 2,2-dithienylglycolate 1a and methyl 2,3dithienylglycolate $\mathbf{1 b}$ synthesis. Depending on both the nature of the bromothiophene derivative used and the condensation conditions, it was possible to obtain either $1 \mathbf{a}$ - the key starting material in the preparation of important anticholinergic agents - or $\mathbf{1 b}$, precursor of pharmacological impurities. By this way $\mathbf{1 b}$ was fully characterized, giving the characteristic signals that permit its differentiation for the target compound.

\section{Experimental Section}

General. All available chemicals and solvents were purchased from commercial sources and were used without any further purification. Thin layer chromatography (TLC) was performed using $0.25 \mathrm{~mm}$ silica gel precoated plates Si 60-F254 (Merck) visualized by UV-254 light and CAM staining. Purification by flash column chromatography (FCC) was conducted by using silica gel Si 60, 230-400 mesh, 0.040-0.063 mm (Merck). 
Melting points were determined on a Büchi B450 apparatus and are corrected. ${ }^{1} \mathrm{H}$ and ${ }^{13} \mathrm{C}$ NMR spectra were recorded on a Bruker Fourier 300 (recorded at: $300.13 \mathrm{MHz}$ for ${ }^{1} \mathrm{H} ; 75.00 \mathrm{MHz}$ for ${ }^{13} \mathrm{C}$ ) or Bruker Avance Spectrometer (recorded at: $400.13 \mathrm{MHz}$ for ${ }^{1} \mathrm{H} ; 100.62 \mathrm{MHz}$ for ${ }^{13} \mathrm{C}$ ); chemical shifts are indicated in ppm downfield from TMS, using the residual proton $\left(\mathrm{CHCl}_{3} 7.28 \mathrm{ppm}\right.$; acetone $\left.2.05 \mathrm{ppm}\right)$ and carbon $\left(\mathrm{CDCl}_{3} 77.0\right.$ ppm; acetone 207.1 and 30.9 ppm) solvent resonances as internal reference. Coupling constants values $J$ are given in $\mathrm{Hz}$.

Preparation of methyl 2-oxo-2-(thiophen-3-yl)acetate (4b). In a flame-dried round flask, BuLi [1.6] (0.60 mL, $0.95 \mathrm{mmol}$ ) was added by syringe under $\mathrm{N}_{2}$ to a solution of dimethyl oxalate (177 $\left.\mathrm{mg}, 1.5 \mathrm{mmol}\right)$ and 3bromothiophene $(193 \mathrm{mg}, 1.0 \mathrm{mmol})$ in anhydrous THF $(25 \mathrm{~mL})$ at $-80{ }^{\circ} \mathrm{C}$. The reaction mixture was stirred at $80{ }^{\circ} \mathrm{C}$ until completion [30 min, TLC analysis - AcOEt/hexane (1:9)], then was quenched with saturated $\mathrm{NH}_{4} \mathrm{Cl}$ solution $(5 \mathrm{~mL})$. After extraction with AcOEt $(2 \times 20 \mathrm{~mL})$, the collected organic phases were washed with brine $(1 \times 10 \mathrm{~mL})$, dried over $\mathrm{Na}_{2} \mathrm{SO}_{4}$ and, after evaporation of the solvent in vacuum, the crude was purified by FCC AcOEt/hexane (1:9) - on silica gel to afford the pure compound $\mathbf{4 b}(112 \mathrm{mg}, 66 \%)$ as a slightly yellow waxy solid; ${ }^{1} \mathrm{H}$ NMR (400 MHz, Acetone- $\left.d_{6}\right) \delta 8.67(\mathrm{dd}, 1 \mathrm{H}, J 2.7,1.5 \mathrm{~Hz}), 7.67-7.65(\mathrm{~m}, 2 \mathrm{H}), 3.96(\mathrm{~s}, 3 \mathrm{H}) ;{ }^{13} \mathrm{C} \mathrm{NMR}$ $\left(75 \mathrm{MHz}\right.$, Acetone- $\left.d_{6}\right) \delta 179.3,164.0,138.7,138.2,128.3,127.7,52.9$. Anal. Calcd. for $\mathrm{C}_{7} \mathrm{H}_{6} \mathrm{O}_{3} \mathrm{~S}: \mathrm{C}, 49.40 ; \mathrm{H}$, 3.55. Found: $\mathrm{C}, 49.01 ; \mathrm{H}, 3.48$.

Lithiation of 2-Bromothiophene: preparation of oxo-acetate $(4 a, c)$ and glycolates $(1 a, b)$. In a flame-dried round flask, organolithium reagent $(0.95 \mathrm{mmol})$ was added dropwise to a solution of 2-bromothiophene $\mathbf{1 b}$ (193 $\mathrm{mg}, 1.0 \mathrm{mmol})$ in anhydrous THF $(15 \mathrm{~mL})$ under $\mathrm{N}_{2}$ at $-80^{\circ} \mathrm{C}$. After $20 \mathrm{~min}$, a THF $(5.0 \mathrm{~mL})$ solution of oxalate 3 or oxo-acetate $4 \mathbf{a}-\mathbf{c}$ was added. After the disappearing of the starting thiophene, the reaction was quenched by saturated aqueous $\mathrm{NH}_{4} \mathrm{Cl}$ and extracted with AcOEt $(2 \times 20 \mathrm{~mL})$; the collected organic phases were washed with brine $(1 \times 10 \mathrm{~mL})$, dried over $\mathrm{Na}_{2} \mathrm{SO}_{4}$ and the solvent was evaporated under vacuum (RV). The resulting crude was purified by FCC - AcOEt/hexane (1:9) - on silica gel. Yield, physical, spectroscopic and analytical data of products $\mathbf{4 a , c}, \mathbf{1 a}, \mathbf{b}$ are as follows.

Methyl 2-oxo-2-(thiophen-3-yl)acetate 4a. BuLi [1.6] (0.6 mL), dimethyl oxalate 3 (177 mg, $1.5 \mathrm{mmol}) .4 a(124$ $\mathrm{mg}, 73 \%, 40 \mathrm{~min}$ ); slightly yellow waxy solid. ${ }^{1} \mathrm{H} \mathrm{NMR}\left(300 \mathrm{MHz}, \mathrm{CDCl}_{3}\right) \delta 8.18$ (dd, $\left.1 \mathrm{H}, J 3.9,1.1 \mathrm{~Hz}\right), 7.84$ (dd, $1 \mathrm{H}, J$ 4.9, $1.1 \mathrm{~Hz}$ ), 7.22 (dd, 1H, J 4.9, $3.9 \mathrm{~Hz}), 3.99(\mathrm{~s}, 3 \mathrm{H}) ;{ }^{13} \mathrm{C} \mathrm{NMR}\left(75 \mathrm{MHz}, \mathrm{CDCl}_{3}\right) \delta 175.9,162.0,139.0$, 137.6, 137.4, 128.7, 53.2. Anal. Calcd. for $\mathrm{C}_{7} \mathrm{H}_{6} \mathrm{O}_{3} \mathrm{~S}: \mathrm{C}, 49.40 ; \mathrm{H}, 3.55$. Found: $\mathrm{C}, 49.01 ; \mathrm{H}, 3.51$.

Methyl 2-(3-bromothiophen-2-yl)-2-oxoacetate (4c). LDA [1.0] (0.95 mL), dimethyl oxalate 2 (177 mg, 1.5 mmol). 4c (90 mg, 36\%, $30 \mathrm{~min})$; yellow solid. ${ }^{1} \mathrm{H}$ NMR $\left(300 \mathrm{MHz}, \mathrm{CDCl}_{3}\right) \delta 7.94(\mathrm{~d}, 1 \mathrm{H}, J 4.2 \mathrm{~Hz}), 7.19$ (d, $1 \mathrm{H}, J$ $4.2 \mathrm{~Hz}), 3.99$ (s, 3H); ${ }^{13} \mathrm{C} \mathrm{NMR}\left(75 \mathrm{MHz}, \mathrm{CDCl}_{3}\right) \delta 174.1,161.4,139.8,137.6,131.8,127.4$, 53.4. Anal. Calcd. for $\mathrm{C}_{7} \mathrm{H}_{5} \mathrm{BrO}_{3} \mathrm{~S}$ : C, 33.75; $\mathrm{H}, 2.02$. Found: $\mathrm{C}, 33.39 ; \mathrm{H}, 1.99$.

Methyl 2-hydroxy-2-(thiophen-2-yl)-2-(thiophen-2-yl)acetate (1a). BuLi [1.6] (0.6 mL), oxo acetate 4a (187 $\mathrm{mg}, 1.1 \mathrm{mmol}) .1 \mathrm{a}(191 \mathrm{mg}, 75 \%, 30 \mathrm{~min})$, whitish solid, mp $94-95^{\circ} \mathrm{C} .{ }^{1} \mathrm{H} \mathrm{NMR}\left(300 \mathrm{MHz}, \mathrm{CDCl}_{3}\right) \delta 7.32(\mathrm{~d}, 2 \mathrm{H}$, J $4.9 \mathrm{~Hz}$ ), $7.20-7.18(\mathrm{~m}, 2 \mathrm{H}), 7.00(\mathrm{dd}, 2 \mathrm{H}, J 5.1,3.6 \mathrm{~Hz}), 4.68(\mathrm{~s}, 1 \mathrm{H}), 3.92(\mathrm{~s}, 3 \mathrm{H}) ;{ }^{13} \mathrm{C} \mathrm{NMR}\left(75 \mathrm{MHz}^{\mathrm{CDCl}} \mathrm{CD}_{3} \delta\right.$ 172.3, $145.7\left(2 \mathrm{C}_{\mathrm{Ar}}\right), 126.8\left(2 \mathrm{CH}_{\mathrm{Ar}}\right), 126.0\left(2 \mathrm{CH}_{\mathrm{Ar}}\right), 125.9\left(2 \mathrm{CH}_{\mathrm{Ar}}\right), 76.4,54.3$. HPLC analyses were carried out on a Zorbax Rx-C8 column $\left(5 \mu \mathrm{m}, 4.6 \times 150 \mathrm{~mm}\right.$ ) by using as eluent a mixture of solvents [solvent $\mathrm{A} \mathrm{H}_{2} \mathrm{O}$ (with $1 \%$ trimethylamine and adjusting $\mathrm{pH}$ to 3.0 with perchloric acid) and solvent $\mathrm{B} \mathrm{CH}_{3} \mathrm{CN}$ ]. Gradient: $0-20 \mathrm{~min}, \% \mathrm{~B}$ 25 (isocratic); 20 - $36 \mathrm{~min}, \%$ B 51 (gradient); $36-36.1 \mathrm{~min}, \%$ B 25 (gradient). Flow rate $2 \mathrm{~mL} / \mathrm{min}, \mathrm{T}=25^{\circ} \mathrm{C} ; \mathrm{UV}$ detector $\lambda 254 \mathrm{~nm}$. Retention times: 1b, $13.20 \mathrm{~min} ; 1 \mathrm{a}, 14.51 \mathrm{~min}$. Anal. Calcd. for $\mathrm{C}_{11} \mathrm{H}_{10} \mathrm{O}_{3} \mathrm{~S}_{2}: \mathrm{C}, 51.95 ; \mathrm{H}$, 3.96. Found: $\mathrm{C}, 51.71 ; \mathrm{H}, 3.89$.

Methyl 2-hydroxy-2-(thiophen-2-yl)-2-(thiophen-3-yl)acetate (1b). BuLi [1.6] (0.6 mL), oxo acetate 4b (187 $\mathrm{mg}, 1.1 \mathrm{mmol}) .1 \mathrm{~b}(173 \mathrm{mg}, 68 \%, 30 \mathrm{~min})$, greyish waxy solid, $\mathrm{mp} 90-92{ }^{\circ} \mathrm{C} .{ }^{1} \mathrm{H} \mathrm{NMR}\left(400 \mathrm{MHz}\right.$, Acetone- $\left.\mathrm{d}_{6}\right) \delta$ 
7.47 (s, 1H), 7.44 (dd, 1H, J 5.1, 3.2 Hz), 7.41 (dd, 1H, J 5.2, 1.2 Hz), 7.22 (dd, 1H, J 5.0, 1.4 Hz), 7.12 (dd, $1 \mathrm{H}, J$ 3.6, $1.2 \mathrm{~Hz}$ ), 7.00 (dd, $1 \mathrm{H}, J 5.1,3.6 \mathrm{~Hz}), 5.76$ (bs, $1 \mathrm{H}), 3.83(\mathrm{~s}, 3 \mathrm{H}) ;{ }^{13} \mathrm{C} \mathrm{NMR}(101 \mathrm{MHz}$, Acetone-d6) $\delta 173.3$, 147.9, 144.7, 127.6, 127.1, 126.2, 126.1, 126.0, 123.2, 77.5, 53.3. Anal. Calcd. for $\mathrm{C}_{11} \mathrm{H}_{10} \mathrm{O}_{3} \mathrm{~S}_{2}$ : C, 51.95; $\mathrm{H}, 3.96$. Found: C, 51.55; H, 3.90 .

\section{Supplementary Material}

Supplementary material related to this article, including Nuclear Magnetic Resonance $\left({ }^{1} \mathrm{H}\right.$ and ${ }^{13} \mathrm{C}$ NMR) figures for methyl dithienyl glycolates $\mathbf{1} \mathbf{a}, \mathbf{b}$ and oxo-acetate $\mathbf{4 a - c}$ are available in the online version of the text.

\section{References}

1. Banholzer, R.; Bauer, R.; Reichl, R. EP 418716 A1 Mar 27, 1991.

2. Banholzer, R.; Graulich, M.; Luettke, S.; Mathes, A.; Meissner, H.; Specht, P.; Broeder, W. DE 10064816 A1 Jun 27, 2002.

3. Scarpitta, F.; Busolli, J.; Diulgheroff, N.; Volonte, R.; Pontiroli, A. WO 2008008376 A2 Jen 17, 2008.

4. Belzer, W.; Hamm, R.; Hofmann, M.; Lock, R. DE 102004041253 A1 Mar 02, 2006.

5. Tengfeng, T.; Liangzhong, L.; Jun, X.; Jin, Q.; Hong, P.; Ping, L.; Xiaole, L. CN 1861598 A Nov 15, 2006.

6. Xiaole, L. (2008), CN 101230063 A Jul 30, 2008.

7. Zhengyi, M.; Xinhua, L.; Xiaole, L. CN 101768158 A Jul 07, 2010.

8. Kadam, V. T.; Edaki, D. U.; Oruganti, N.; Minhas, H. S.; Minhas, G. S. WO 2016162878 A1 Oct 13, 2016; see also Refs. 1 and 4.

9. Xiang, Z.; Liu, J.; Sun, H.; Wen, X. ChemMedChem, 2017, 12 (15), 1173-1182. https://doi.org/10.1002/cmdc.201700189

Sun, H.; Xiang, Z.; Wen, X. CN 103965178 A Aug 06, 2014.

10. Sun, H.; Wen, X.; Liu, J.; Xiang, Z. CN 107213141 A Sep 29, 2017.

11. Collingwood, S. P.; Cox, B.; Baettig, U.; Bhalay, G.; Devereux, N. J. WO 2004096800 A2 Nov 11, 2004.

12. Collingwood, S. P.; Baettig, U.; McCarthy, C. WO 2005000815 A2 Gen 06, 2005.

13. Ray, N. C.; Van Neil, M. B. WO 2010071581 A1 Jun 24, 2010.

14. Collingwood, S. P.; Baettig, U.; Mccarthy, C. US 7947730 B2 May 24, 2011.

15. Prat, M.; Fernández; D.; Buil, M. A.; Crespo, M. I.; Casals, G.; Ferrer, M.; Tort, L.; Castro, J.; Monleón, J. M.; Gavaldà, A.; Miralpeix, M.; Ramos, I.; Doménech, T.; Vilella, D.; Antón, F.; Huerta, J. M.; Espinosa, S.; López, M.; Sentellas, S.; González, M.; Albertí, J.; Segarra, V.; Cárdenas, A.; Beleta J.; Ryder H. J. Med. Chem., 2009, 52 (16), 5076-5092. https://doi.org/10.1021/jm900132z

16. Issa, F.; Kassiou, M.; Chan, H. K.; McLeod, M. D. Aust. J. Chem. 2006, 59 (1), 53-58. https://doi.org/10.1071/CH05303

17. Thirumalai, R. S.; Sajja, E. IN 2014CH05611 A Jul 01, 2016.

18. Gu, G.; Yang, T.; Lu, J.; Wen, J.; Dang, L.; Zhang, X. Org. Chem. Front. 2018, 5(7), 1209 - 1212 https://doi.org/10.1039/C8Q000047F

19. Li, W.; Han, Y.; Chen, J. Tetrahedron 2017, 73(39), 5813 - 5819 https://doi.org/10.1016/i.tet.2017.08.035 
20. Li, W.; Liu, Y.; Chen, J. . Tet. Lett. 2015, 56(29), 4328 - 4330; https://doi.org/10.1016/j.tetlet.2015.05.073

21. Nicolle, S. M.; Moody, C. J. Chem. Eur. J. 2014, $20(15), 4420$ - 4425. https://doi.org/10.1002/chem.201304656

22. Hayoz, P.; Dueggeli, M. WO 2013050401 A2 Apr 11, 2013.

23. Salman, M.; Kumar, N.; Kaur, K.; Aeron, S.; Sarma, P. K. S.; Dharmarajan, S.; Mehta, A.; Chugh, A. IN 2005DN01810 A Oct 09, 2009.

24. Kumar, N.; Sattigeri, J. A.; Aeron, S.; Garg, M.; Ray, A.; Gupta, S.; Malhotra, S.; Shirumalla, R. K. EP 2130830 A1 Dec 09, 2009

25. Meng, Q.; Sun, Y.; Ratovelomanana-Vidal, V.; Genet, J. P.; Zhang, Z. J. Org. Chem. 2008, 73(10), 3842 3847.

https://doi.org/10.1021/jo800228e

26. Salman, M.; Kumar, N.; Kaur, K.; Aeron, S.; Sarma, P. K. S.; Dharmarajan, S.; Mehta, A.; Chugh, A. WO 2006117754 A1 Nov 09, 2006.

27. Fabrichnyi, B. P.; Stoyanovich, F. M.; Vol'kenshtein, Yu. B.; Taits, S. Z.; Gol'dfarb, Ya. L.; Mezentsev, A. S.; Panina, M. A.; Karmanova, I. B.; Koval'skaya, V. A. SU 677331 A1 Jul 07, 1981.

28. Blicke, F. F.; Tsao, M. U. J. Am. Chem. Soc. 1944, 66, 1645-8. https://doi.org/10.1021/ja01238a011

29. Nyberg, K.; Ostman, B.; Wallerberg, G. Acta Chem. Scand. 1970, 24 (5), 1590-1596; see also Refs. 1 - 18.

30. Each API's impurity greater than $0.1 \%$ must be unambiguously identified for Analytical Method Development and Validation. Guidance for Industry - ANDAs: Impurities in Drug Substances; U.S. Department of Health and Human Services Food and Drug Administration Center for Drug Evaluation and Research (CDER); June 2009

31. Reinecke, M. G.; Adickes, H. W.; Pyun, C. J. Org. Chem., 1971, 18 (36), 2690-2692. https://doi.org/10.1021/jo00817a025

32. Kano, S.; Yuasa, Y.; Yokomatsu, T.; Shibuya, S. Heterocycles 1983, 20, 2035-2037. https://doi.org/10.3987/R-1983-10-2035

33. De Meijere, A.; Zhao, L.; Belov, V. N.; Bossi, M.; Noltemeyer, M.; Hell, S. W. Chem. Eur. J., 2007, 13 (9), 2503-2516.

https://doi.org/10.1002/chem.200601316

34. Okano, K.; Sunahara, K.; Yamane, Y.; Hayashi, Y.; Mori, A. Chem. Eur. J., 2016, 22 (46), 16450-16454. https://doi.org/10.1002/chem.200601316

35. Shono, K.; Sumino, Y.; Tanaka, S.; Tamba S.; Mori A. Org. Chem. Front., 2014, 1, 678-682; Hayashi, Y.; Okano, K.; Mori, A. Org. Lett. 2018, 20, 958-961. 